\title{
Systematic review of animal research $^{1}$
}

\author{
Revisão sistemática da pesquisa em animais
}

\section{Carlos Alberto Guimarães ${ }^{\mathrm{I}}$}

${ }^{\text {I }}$ Full Professor, Serra dos Órgãos University Center (UNIFESO). Associate Professor, Department of Surgery, School of Medicine, Federal University of Rio de Janeiro, Brazil.

${ }^{1}$ Rio systematic review study group.

Systematic review is a review that has been prepared using a systematic approach to minimising biases and random errors which is documented in a methods section, thus providing more reliable findings from which conclusions can be drawn., ${ }^{1,2}$

The characteristics of a systematic review are: a. A set of objectives with eligibility criteria for studies; b. A reproducible methodology; c. A systematic search that attempts to identify all studies that would meet the eligibility criteria; $d$. An assessment of the validity of the results of the included studies; and e. A systematic presentation, and synthesis, of the characteristics and findings of the included studies. ${ }^{2}$

A systematic review may, or may not, include a meta-analysis: the use of statistical methods to summarize the results of independent studies, so providing more precise estimates of the effects of health care than those derived from the individual studies. $^{2}$

Systematic reviews should be viewed as observational studies of the evidence. There are several steps in conducting a systematic review: 1. Formulate review question; 2. Define inclusion and exclusion criteria; 3. Locate studies; 4. Select studies; 5. Assess study quality; 6. Extract data; 7. Analyse and present results; and 8 . Interpret results. ${ }^{3}$

Systematic reviews may provide the highest level of evidence when performed for randomized controlled trials (therapy/prevention/aetiology/harm questions), for cohort studies (prognosis/differential diagnosis/prevalence questions), for diagnosis studies (diagnosis questions), and for economic studies (economic and decision analyses questions). ${ }^{4}$

And what about systematic reviews of animal research? Animal models are often used to test a drug or procedure before proceeding to clinical trials. One reason for use of animal models is that they allow researchers to focus on particular pathological processes. However, it is essential that their results are valid. Imprecise results from animal experiments may result in clinical trials of inert or even harmful substances, thus exposing patients to unnecessary risk. Moreover, if animal experiments fail to inform medical research then the animals suffer unnecessarily. ${ }^{5}$

Systematic reviews of animal research allow a more objective appraisal of the evidence, and systematic reviews across species would help determine whether the results could be generalized to humans. ${ }^{5}$
Systematic reviews is a tool in translation of basic sciences research from laboratory to human research. Attempted systematic reviews of bench studies have a higher proportion of methodological weaknesses than those of whole animal studies, though the latter are not as good as systematic reviews of human clinical trials. ${ }^{6}$

Systematic reviews of animal experiments are uncommon. About 1 in 1000 Medline records pertaining to human research is tagged as a systematic review compared with 1 in 10000 records pertaining to animal research. ${ }^{5}$

In clinical trials, bias is avoided by ensuring strict randomisation with concealed treatment allocation. The extent to which inadequate concealment of allocation might introduce bias in animal experiments is uncertain. However, it is easy to imagine how bias could arise. For example, weaker animals may be easier to catch than healthy animals, and this could result in systematic differences between the intervention and control groups on baseline prognostic factors. ${ }^{5}$

Random error in clinical trials is minimised by increasing the number of participants. However, animal researchers are encouraged to reduce the number of experimental animals to a minimum. As a result, some animal experiments provide little reliable information. Meta-analyses of the results of animal research would increase the precision of estimates of treatment effects. $^{5}$

Publication bias may be a threat to validity in systematic reviews of animal experiments. When searching databases for reviews of animal experiments, we discover that the majority of the work undertaken is kept confidential. So, systematic reviews are likely to be flawed. The proportion of the work that gets published in a form that is available to the public is unknown. Special efforts (contact with experts, laboratories and related research associations) will be needed to retrieve unpublished data. This is one of most important challenges for reviewers of animal studies. ${ }^{6}$

Prospective registration of animal experiments may help to avoid the problem of publication bias. ${ }^{5}$

Systematic reviews of animal research could include a range of animal species. If the results were consistent across species this would indicate that they might also apply in humans, and this would be valuable information. ${ }^{5}$ 
Systematic reviews of animal experiments would help to ensure that animal experiments do not set out to answer questions that have already been answered, reduce bias and increase precision, and provide reassurance about whether the results can be generalised. On methodological grounds, animal experimentation would better contribute to human health care if we promoted registration, randomization, and systematic reviews. ${ }^{5}$

In conclusion, there is a need for rigour when reviewing animal research. The editorial Board of Acta Cirúrgica Brasileira is working on the organization of a special committee for analysis of systematic reviews that address a topic of current interest in surgical practice.

\section{References}

1. Egger M, Smith GD, O`Rourke K. Systematic Reviews in Health Care: Meta-analysis in Context. 2nd ed. London: BMJ Books; c2006. Chapter 1, Rationale, potentials, and promise of systematic reviews; p.3-19.
2. Green S, Higgins JP, Alderson P, Clarke M, Mulrow CD, Oxman AD. Cochrane Handbook for Systematic Reviews of Interventions Version 5.0.0 [Internet]. London: The Cochrane Collaboration; 2008 Feb [cited 2008 Sep 14]. Chapter 1, Introduction. Available from: http://www.cochrane-handbook.org/

3. Egger M, Smith GD. Systematic Reviews in Health Care: Metaanalysis in Context. 2nd ed. London: BMJ Books; c2006. Chapter 2, Principles of and procedures for systematic reviews; p.23-42.

4. Centre for Evidence-Based Medicine [Internet]. Oxford: CEBM. Levels of Evidence [cited 2008 Sep 14]; [about 3 screens]. Available from: http://www.cebm.net/index.aspx?o=1025

5. Roberts I, Kwan I, Evans P, Haig S. Does animal experimentation inform human healthcare? Observations from a systematic review of international animal experiments on fluid resuscitation. BMJ. $2002 \mathrm{Feb}$ 23;324:474-6.

6. Mignini LE, Khan KS. Methodological quality of systematic reviews of animal studies: a survey of reviews of basic research. BMC Medical Research Methodology. 2006;6:10. doi:10.1186/1471-2288-6-10.

7. Matos D, Lustosa SA. Surgical practice: is it evidence-based? [editorial]. Acta Cir Bras. 2008;23(2):115-7.

\section{Correspondence:}

Carlos Alberto Guimarães

Rua Luís Murat, 180

25959-060 Teresópolis - RJ Brazil

GERS-Rio@yahoogroups.com

http://tech.groups.yahoo.com/group/GERS-Rio

\section{How to cite this article}

Guimarães CA. Systematic review of animal research. Acta Cir Bras. [serial on the Internet] 2009 Jan-Feb;24(1). Available from URL: http://www.scielo.br/acb 